\title{
A Survey on Current Technologies for Web Development
}

\author{
A. V. Akhil Krishna ${ }^{1}$ \\ ${ }^{1}$ Student, \\ Dept. of Information Science and Engineering, \\ R.V. College of Engineering, \\ Bengaluru, India
}

\author{
Dr. Padmashree $\mathrm{T}^{2}$ \\ ${ }^{2}$ Assistant Professor, \\ Dept. of Information Science and Engineering, \\ R.V. College of Engineering, \\ Bengaluru, India
}

\begin{abstract}
Web-based application developers are presented with a dizzying variety of option formats, languages, frameworks and technological objects. We survey, identify and compare technologies that help the creation of Web apps. We conclude that, while the Web's connectivity problems have been largely solved, the cacophony of Web-based applications technologies represents the lack of a solid model tailored to this domain.
\end{abstract}

Key Words: Technology, NoSQL, MySQL, Angular, NodeJS

\section{INTRODUCTION}

The world has changed to a mobile era, but still today no app could be as effective as web-based applications. The prevalence of progressive web apps which perform functions identical to mobile apps is surfacing on top of this. So, it's pretty clear that web apps will remain and continue for a long time to come. What makes this web applications worth investing in is the use for development of high-end scalable technologies or in simpler words, using state-of-the-art quality stack technology.

In addition, the business penetration and performance of the application can be maximized with the most suitable technology stack. The use of a poor technology stack could also lead to a failure of the entire project. Therefore, it is important to have a thorough look at the web development stack that will be used for the development of a project.

\section{DIFFERENT SOLUTIONS}

This section reviews various technologies currently available for web development

1. In [1] this paper it has presented a comparative study of non-relational databases and relational databases. It mainly focuses on presentation on one implementation of the NoSQL database technology, namely MongoDB, and draws a comparison with MYSQL which is another database and tries to justify why MySQL is less preferable and less efficient than MongoDB. It also discusses the disadvantages of using a relational database compared to a non-relational database.

The forum is developed using MongoDB, a NoSQL database chosen from a variety of non-relational databases. The database integration in the framework is also be presented. If the application is data-intensive and stores many data and queries, we can choose MongoDB instead of
MySQL. It should also be noted that in certain situations, each user requires his or her own individual settings in the same application and connection database does not permit a full customization based only on the user's needs. Therefore, increasingly more applications start using a nonrelational database, as they provide a more versatile framework that can be formed according to the needs of each user.

2. The advantages of using AngularJS to create the front-end framework, NodeJS to design the Back-end Web server and the performance benefits of storing data based on MongoDB are discussed in [2] this paper. This paper concentrates on MongoDB storage solutions for the storage of large data and on MapReduce-based analysis solutions. This paper discusses on how Web services are designed that have the requirements of large data visualization based on NodeJS. Within this paper, the design of the express monitoring system is implemented. The primary technologies used in system design was first discussed. It also primarily introduces the framework architecture, MongoDB database-based storage solutions and the MapReduce-based analysis solutions. Finally, this paper introduces the Express Supervision Framework interface application and statistical analysis modules. Machine learning is to be used in future.

3. In [3] this paper, it characterizes the runtime behaviors on an evolving server-side application JavaScript, Node.js, compared to JavaScript's client-side code. The runtime profile reveals that the V8 C++ library used for server load is spent a considerable amount ( $47.5 \%$ on average) of the total CPU time, while it is only $3.2 \%$ on the client-side.

The study on the server-side system of complex call contexts shows that function calls to the V8 runtime to handle JavaScript objects lead significantly towards high CPU utilization in the V8 library. We focused on the overuse of the V8 library code with CPUs and analyzed the call stacks in detail during the execution of the library code. The function executes the $\mathrm{C}++$ code of the serverside JavaScript framework which implements the node.js API, and native code in the package node module is one reason for the excess of the CPU use in the V8 library.

4. In [4] this paper discusses the latest developments which received popularity in the server-side JavaScript space, 
Node.js. It's a framework for designing high-performance, concurrent programs that are based on asynchronous I/O and event driven programming model and not the multithreading approach.

As we are aware that JavaScript knowledge is essential for exceptional UI interactions, the idea of using one programming language for everything becomes quite tempting. The architecture of Node.js makes it easy to use a functional and highly meaningful language for server programming, without any performance issues and stepping out of the programming mainstream.

5. In [5] this paper few possible security measures are outlined to be aware of when using Node.js platform and server-side JavaScript. It also identifies two vulnerabilities and provides recommendations for the development and configuration of secure and resilient Node.js web applications.

It analyzed the security implications of the architecture and technologies used in the Node.js platform. Two new denial of service vulnerabilities in the platform are discussed in the paper. Its analysis showed that while the platform is suitable for production use for some applications, it should be avoided in security critical applications. This is because the model is immature and other safety flaws are inherent. Security-conscious programming will avoid many of the problems we have highlighted. However, errors with Node.js applications are much more common as programmers lack extensive experience in writing longstanding server-side JavaScript asynchronous applications.

6. In [6] this paper discusses the issues of utilizing the Aspect-Oriented Programming concept, and demonstrates this approach through Angular 2, a JavaScript framework and compares it with the traditional development approach.

The new Angular framework develops better programming paradigms. TypeScript with type control and support for ANGULAR annotations provides a high degree of language sensation to the customer program. This will however increase the volume of code and restatement of information. This article addresses the library and makes form creation a better experience in this context. Easy library usage enables programmers to specify shape entries to a server application, when the entries are serialized into JSON and transmitted to the Angular. The rendering is carried out in the dynamic forms. Writing the code is easier, looks more comfortable and is much more durable. This is especially noteworthy when it comes to multiple types or supports context-aware user interfaces. The study shows an impact on reading, the volume of code and maintenance efforts for fragments in practice.

7. In [7] In this paper, the specific characteristic traits of MongoDB and the design principles were studied and based on the analysis an anti-normal form model was proposed. This method is based on the theory of data dependency and uses a breakdown or joint data strategy to develop the logic schema of non-related databases for different dependencies.
This article gives the application of MongoDB in BBS information collection system. The data storage structure and queries are designed, and the advantages and disadvantages of data structure, query and scalability are listed. Relational database contains several tables with external keys, a sharp decrease in query efficiency with a lot of data and high cost vertical scalability.

8. In this paper [8] a pragmatic study was performed to understand how JavaScript developers make use of various language features and assist future extensions of JavaScript. The study displays that there is a clear uncertainty about newly introduced features. Tool developers can study the requirement for better IDEs and tools and programmers can study about the good parts of JavaScript.

9. In this paper [9] it addresses the test and difficulty of the scalability, versatility and processing capacities of traditional relational database management systems (RDBMS) by incorporating hierarchical, semi-structural and unstructured data. The new frameworks require the databases to be scaled horizontally. The data in the databases must be stored and processed in various formats, including unstructured data. These questions are answered by NoSQL methodologies. The conventional approach to relationship and objective-driven DBMS can't adapt to today's systems with the flexible scaling necessary. Similarly, large databases have been created. But specialized technologies that can support these databases have not emerged. These massive repositories can't be preserved in a single physical device. A collaborative system for this is recommended. A NoSQL framework can be used with a particular objective to improve unstructured data processing. To investigate the merits of NoSQL databases, a NoSQL database, MongoDB which is document oriented, that typically uses JSON data is discussed. An administrative tool has been developed in the research to evaluate the MongoDB database scheme. There are two algorithms: one for local analysis and the other for aggregate analysis. Automated testing and real users tested the created solution. The solution was calculated to be quicker than the solutions already available.

10. The aim of the paper [10] was to create a web administration interface for the MongoDB database to conduct analysis on the stored data. The needs of potential users and solutions similar that already existed were taken into accounts and the application was designed. The tool is composed of a console application that analyzes the results and a web application that visualizes the results. Console application can also be used and integrated into other tools separately. The app is quicker than existing solutions, more feature-rich and automatic testing and actual user testing have been performed.

The system also has a solution for processing 3D parcels. It also proposes the use of Robots empowered with computer vision in material handling. Autonomous Guided Vehicles 
which is a flexible solution are based on active sensors for self-orientation. This is seen as the best solution that removes most of the manual efforts in station loading and unloading and also in material handling with the premises.

\section{REFERENCES}

[1] Cornelia Györödi, Robert Györödi, George Pecherle , Andrada Olah," A comparative study: MongoDB vs. MySQL", 2015 13th International Conference on Engineering of Modern Electric Systems (EMES)

[2] Li Liang, Ligu Zhu, Wenqian Shang, Dongyu Feng, Zida Xiao, "Express supervision system based on NodeJS and MongoDB", 2017 IEEE/ACIS 16th International Conference on Computer and Information Science (ICIS)

[3] Takeshi Ogasawara, "Workload characterization of server-side JavaScript", 2014 IEEE International Symposium on Workload Characterization (IISWC)

[4] Stefan Tilkov; Steve Vinoski, "Node.js: Using JavaScript to Build High-Performance Network Programs" , 2010 IEEE Internet Computing

[5] Andres Ojamaa ; Karl Düüna, "Assessing the security of Node.js platform" 2012 International Conference for Internet Technology and Secured Transactions

[6] Zdenek Brabec ; Tomas Cerny ; Jiri Sebek, "On Metadata Extension to Derive Data Presentations with Angular 2", 2016 6th International Conference on IT Convergence and Security (ICITCS)

[7] Yunhua Gu; Shu Shen; Jin Wang ; Jeong-Uk Kim, "Application of NoSQL database MongoDB" 2015 IEEE International Conference on Consumer Electronics - Taiwan

[8] Sharath Gude, Munawar Hafiz, Allen Wirfs-Brock, "JavaScript: The Used Parts", IEEE $38^{\text {th }}$ Annual Computer Software and Applications Conference 2014

[9] Benymol Jose, Sajimon Abraham, "Exploring the merits of nosql: A study based on mongodb" 2017 International Conference on Networks \& Advances in Computational Technologies (NetACT)

[10] Liberies Vokorokos , "MongoDB scheme analysis" 2017 IEEE 21st International Conference on Intelligent Engineering Systems (INES) 